\title{
Immune Markers in Non-Small Cell Lung Cancer: What Else Beyond Pd-L1 and Tumour Mutational Burden is there?
}

\author{
JB Jaroslav Bublevič ${ }^{* 1,2}$, SC Saulius Cicėnas ${ }^{3,4}$ and VP Vita Pašukoniene் ${ }^{5}$ \\ ${ }^{1}$ Chemotherapy department, National cancer institute of Lithuania, Lithuania \\ ${ }^{2}$ Pathology, pharmacology and forensic medicine department, Medical faculty, Vilnius University, Lithuania
}

${ }^{3}$ Department of thoracic surgery, Lithuania

${ }^{4}$ Health sciences institute, Lithuania

${ }^{5}$ Immunology department, National cancer institute of Lithuania, Lithuania

Received: 眥: December 31, 2018; Published: 跙: January 09, 2019

*Corresponding author: JB Jaroslav Bublevič, Pathology, pharmacology and forensic medicine department, Medical faculty, Vilnius

University, Lithuania

\section{Abstract}

The advancement of immune-oncology and immunotherapy in recent decades is unprecedented. Immune evasion and cancer promoting inflammation have been added as new hallmarks of malignancy. This opened a view of cancer as an immunological disease. Non-small cell lung cancer [NSCLC] is one of the leading cancer types in terms of immunotherapy research and implication. The need for predictive markers to immune checkpoint inhibitors fuels the search for immune markers in NSCLC. However, since the importance of immune evasion is now recognised, defining the immunological phenotype of NSCLC can have broader implications. Immune markers may aid in better defining the prognosis, supplement TNM staging and predict response to all modalities of treatment. PD-L1 and TMB are immune markers firmly established in clinical practice. However, 2 markers can hardly capture the full scope of immune response in cancer. We have searched PubMed, Cochrane and clinicaltrials.gov databases to compile a brief review of immune markers that seem closest to daily clinical practice in NSCLC other than PD-L1 and TMB. Our aim is to review the possible implications of these markers in personalization of treatment of NSCLC patients. The immune markers helpful in defining prognosis, staging and predicting response to various treatment modalities.

Keywords: Tumour Infiltrating Lymphocytes; Tumour Associated Macrophages; Immuno Oncology; Biomarkers; NSCLC; Personalization

Abbreviations: NSCLC: Non-Small Cell Lung Cancer; ICI: Immune Checkpoint Inhibitors; TMB: Tumour Mutational Burden; TAMS: Tumour Associated Macrophages; DC: Dendritic Cells; MDSC: Myeloid Derived Suppressor Cells; TNBC: Triple Negative Breast Cancer; TILs: Triple Negative Breast Cancer; DFS: Disease-Free Survival; RT: Radio Theraphy; CRC: Colorectal Cancer; DAMP: Damage-Associated Molecular Patterns; APC: Antigen Presenting Cells; TME: Tumour Microenvironment

\section{Introduction}

After being abandoned for the several decades, immuneoncology has since made huge leaps to establish itself as one of the cornerstones of cancer research. The ability to evade the destruction by the immune system has been included as one of the hallmarks of a malignant disease [1]. The complexity of the relationship between the cancer and the immune system should be stressed out. The evolving view of this topic has led to the change of the original immune-surveillance theory into immune-editing [2]. The immune editing theory considers the possibility of selection of less immunogenic clones and the eventual evolution of cancer that allows it to evade or even take advantage of immune systems' elements. The great progress made in this field has resulted in advance of immune checkpoint inhibitors into daily practice. These clinical trials have even placed it as a preferred 1st line treatment in some chemosensitive cancer types. NSCLC with high PD-L1 expression is one such example. However, a considerable number of patients do not benefit from this treatment and long-lasting responses are seen only in few responders. 
The means to immune evasion are many and are not limited to expression of immune checkpoints. The formation and execution of anticancer immune response is described by cancer immunity cycle [3]. Cancer may inactivate the immune response at any step of this cycle: formation of neoantigens, antigen uptake, DC maturation and migration, antigen presentation and T cell priming, T cell migration/ tumour infiltration and finally effector functions of $\mathrm{T}$ cells at the tumour. Based on this, three cancer immune phenotypes have been described: immune-desert, excluded and inflamed tumours [4]. Each phenotype is associated with immune response inactivation at various points of the cancer immunity cycle. Furthermore, every step of this cycle may be impaired by several distinct mechanisms. The identification of the immune phenotype and the underlying mechanisms of immune evasion may therefore be crucial in personalization of therapy.

With immunotherapy taking a strong position in the arsenal against NSCLC, the search for predictive biomarkers for immune checkpoint inhibitors (ICI) continues. However, considering the importance of immune evasion in pathogenesis of cancer, the definition of these mechanisms may also be relevant in respect to other treatment modalities.

Indeed, much evidence shows that various immune factors can predict outcomes after surgery, while radiotherapy and chemotherapy may boost the anticancer immunity by causing immunogenic cell death. PD-L1 and tumour mutational burden [TMB] are immune markers firmly established in clinical practice. However, 2 markers can hardly capture the full scope of immune response in cancer. Therefore, defining the immune microenvironment of NSCLC may be significant in clinical practice beyond predicting response to immunotherapy. In this small nonsystematic review, we attempt to broadly outline feasible immune markers other than PD-L1 and TMB, their limitations and their combinations that may improve the personalized treatment of NSCLC.

\section{Tumour Infiltrating Immune Cells}

Various immune cells infiltrating NSCLC play crucial roles. They both execute the anticancer immune response but can also be recruited by cancer to promote tumour development. Unsurprisingly this has made them the focus of attention as potential biomarkers in NSCLC. Tumour infiltrating lymphocytes [TILs], tumour associated macrophages [TAMs], dendritic cells [DC], myeloid derived suppressor cells [MDSC] and cells of other lineages were researched to various extent. Here we review the mostly researched immune cells in NSCLC. Tumour infiltrating lymphocytes [TILs] are the main effector cells responsible for anti-cancer immunity. Depending on the degree of TIL infiltration, tumours of all types can be classified as cold [low infiltration] or hot tumours [high infiltration]. TILs may be utilized as a prognostic tool widely referred to as immunoscore. An international task force has been established to promote research and integration of immunoscore into routine classification and staging of cancer of all types [5]. Robust evidence supports prognostic value of TILs in triple negative breast cancer [TNBC]. It was shown that increase in intratumoral and stromal lymphocytic infiltrations [in H\&E stains] was associated with reduced risk of relapse and reduced risk of death in TNBC regardless of chemotherapy type [6]. An international group of experts "TIL working group" has formed recommendations for TIL assessment in BC [7].

These guidelines were tested retrospectively on pathologic tissues of 897 TNBC patients. It was established that high TIL density enables to accurately determine patients with favourable disease-free survival [DFS] and overall survival [8]. TILs also predict response to neoadjuvant treatment in TNBC and HER2 positive primary $\mathrm{BC}$, especially to carboplatin containing regimens [9]. The positive prognostic value of TILs was confirmed in other studies as well [10]. Prospective phase III trials in these areas are ongoing. The prognostic role of TILs was demonstrated in colorectal cancer [CRC] as well. Galone et al measured CD3+ and CD8+ TIL density using digital image analysis in the pathologic tissues of 1,336 CRC patients. The authors found that time to recurrence was significantly longer in patients stages I/II/III with high immune infiltration [IM]. Low-IM identified a subgroup of patients with high-risk stage II CRC. The developed assay allows clinicians to more accurately distinguish patients with stage II CRC that are at high risk of recurrent disease [11]. Another study found that the density of CD45RO+ TILs [memory lymphocytes] can predict tumour downstaging and $\gamma$ pTNM stage for rectal cancer following 30 Gy/10f neoadjuvant radiotherapy [RT] and predict better DFS [12] (Figure 1). 


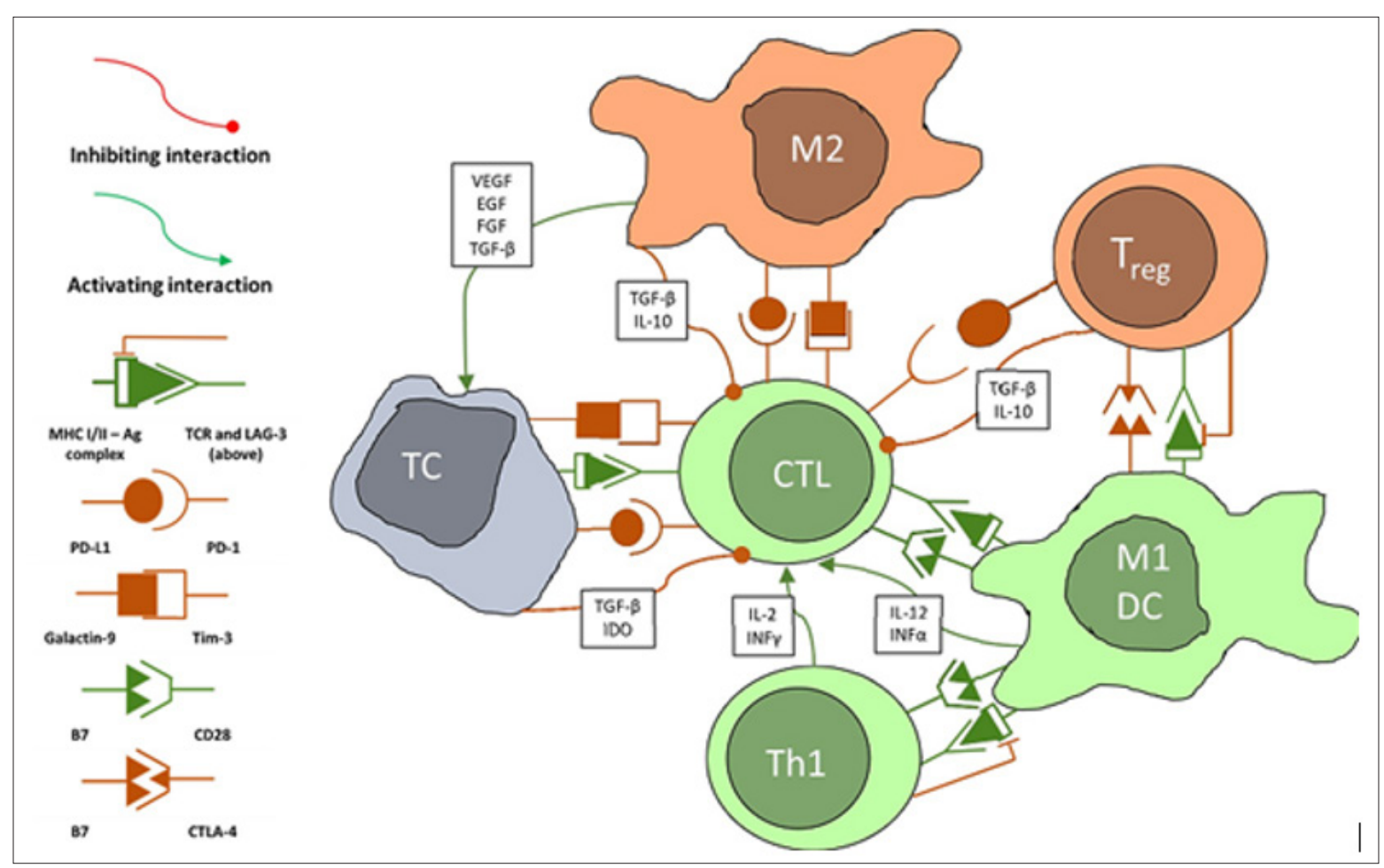

Figure 1: Simplified diagram of interactions between tumour cells (TC) and immune cells. Only relationships between the reviewed cells have been demonstrated. The real scope of interactions is much larger, and some are incompletely understood. Some of the shown interactions take place in the lymph nodes or both lymph nodes and the tumour. These include Ag presentation by dendritic cells or M1 macrophages, cross-presentation and Th1 interactions, Treg interactions. Abbreviations: CTL - cytotoxic lymphocyte (CD8+ effector cell), DC - dendritic cells, EGF - epithelial growth factor, IDO - indoleamine 2,3-dioxygenase, IL - interleukin, INF - interferon, M1 - classically activated macrophage, M2 - alternatively activated macrophage, TC - tumour cell, TGF $\beta$ - transforming growth factor $\beta$, Th1 - type 1 helper lymphocyte, Treg - regulatory lymphocyte, VEGF - vascular endothelial growth factor.

As shown above the most widely used methods of evaluation of TILs in BC and CRC differ significantly. The most appropriate method for NSCLC is yet to be established, but the data from BC and CRC give us a sense of possible directions. The general TIL density assessed in H\&E stains was shown to be a positive prognostic factor in LACEbio collaborative group [13]. This group pooled the results of four large trials that evaluated the benefit of adjuvant chemotherapy versus observation in NSCLC [IALT, ANITA, JBR10 and CALGB]. In this group intense TIL infiltration was associated with increased OS [HR, 0.45; 95\% CI, 0.23 to $0.85 ; \mathrm{P}=.01$ ] and DFS [HR, 0.44; 95\% CI, 0.24 to $0.78 ; \mathrm{P}=.005]$. Intense TIL infiltration was observed in $11 \%$ of squamous cell carcinomas [SCC], $4 \%$ of adenocarcinomas and $3 \%$ of tumours of other histology. Intratumoral and stromal TILs are often assessed separately, because it is thought that they represent different subsets. However, in this study it has been suggested that this may reduce precision due to lack of interobserver reproducibility and so the general TIL density was measured [13]. Because of their different biological roles, detailed analysis of TIL subsets may have further prognostic and predictive benefits. This is discussed below.

T lymphocytes [CD3+] and cytotoxic [CD8+] T cells are the main effector cells in anticancer immune response. Considering their biological role and evidence from studies with CRC, quantification of T cells $\left[\mathrm{CD}^{3+}\right]$ and the subset of cytotoxic T cells [CTL, $\left.\mathrm{CD}^{8+}\right]$ has been the focus of most studies in NSCLC. Donnem et al. [14] have demonstrated prognostic value of $\mathrm{CD}^{8+}$ density for NSCLC stage I-III treated with surgery with or without chemotherapy. The study involved 797 patients in 4 cohorts. Each TNM stage was subdivided into 3 subgroups by stromal CD8+ density. Both stromal CD8 density and pStage were independent prognostic factors for DFS and OS. Dividing each pStage into 3 groups by CD8 density allowed for reliable stratification of survival. Several other studies presented similar evidence $[15,16]$. Paulsen et al. [17] explored the additional benefit of CD45RO+ [memory] TILs. The study found that CD45RO+ density was an independent prognostic factor in a multivariate analysis along with stromal CD8 density. This benefit however was limited to the squamous histology.

Other studies, however, produced conflicting results. Liu et al. [18] in a cohort of 159 NSCLC stage III and IV patients found that the presence of CD3[+], CD4[+], CD8[+], and FOXP3 [+] TILs was not correlated with any clinicopathological features or OS. Nonetheless higher FOXP3 [+]/CD8[+] ratio in tumour sites was an independent factor for poor response to platinum-based chemotherapy in the overall cohort. The available data on TILs was summarized in a 
metanalysis by Geng et al. The results showed that both the stromal or tumour islets and generalized CD8+ density were significant positive prognostic factors in terms of OS and DFS. Similar results were observed for CD3+ infiltration [19]. The conflicting results may arise from the fact that the effectiveness of CTLs depends heavily on their activation state, expression of immune checkpoints and the tumour microenvironment [TME]. Evaluation of immune checkpoint expression or various soluble factors in TME, may add prognostic value. One study found, that high CD3+ density and high levels of intratumoral IL-2 [a crucial cytokine for naïve CD8+ development into effector cells] predicted better prognosis in radically resected NSCLC patients.

Both CD8+ and IL-2 were independent prognostic factors in a multivariate analysis [20]. The interpretation of CD8+ infiltration may also not be so straightforward, as there is evidence that specific subsets of CD8+ cells are also important. A subset of CD8+CD103+ tissue resident memory lymphocytes was found to predict better outcomes in early NSCLC. The density of CD8+CD103+ TILs in tumour tissue was significantly associated with better DFS and OS in univariate analysis, but only with DFS in a multivariate analysis. Moreover, this subset of CTLs had high expression of PD-1 and Tim3 immune checkpoints and were able to destroy autologous tumour cells upon administration of PD-1 antibodies [21]. The priming of TILs by antigen presenting cells [APC] is another important event in cancer immunity. Increased dendritic cell density in tumour tissue leads to over 2-fold increase in the CD3+ and CD8+ TIL density. This increase was found to be limited to adenocarcinomas and tumours with high HGMB1 expression in cancer cells [22]. HGMB1 is a wellknown example of DAMP [damage-associated molecular patterns], that can lead to activation of innate immunity cells through TLR ligation.

The obvious combination includes joint evaluation of TILs and immune checkpoints. Both are influenced by regulatory feedback loops in healthy tissues. PD-L1 may be intrinsically induced by cancer cells or may be an adaptive response to an ongoing immune response by cytotoxic TILs. In both cases tumours would be classified as PD-L1 positive, but PD-L1 expression represents completely different immunological status of the tumour, which will determine response to various treatment modalities. Mazzaschi et al analysed 59 adenocarcinoma patients and 79 SCC patients by evaluating the density of CD3+, CD8+ cells and PD-L1 expression. According to the immune contexture of CD3 infiltrate [positive/negative] and PD-L1 expression [positive/negative], the subjects were divided into 4 groups. All the patients in the study received treatment with nivolumab. The PFS and OS in the type III [PD-L1highCD3low, thought to resemble intrinsic induction of PD-L1] were significantly higher than in the most frequent, type II group [PD-L1lowCD3low, immune ignorance] [25.7 and 35.5 mo. vs 12 and 21.7 mo. Respectively, $\mathrm{p}<0.001$ ] [23]. Independently from immune categories, patients with PD-1 low and high CD8/CD3 ratio had 11 mos. gain in OS $[p<0.01]$ compared to the reverse counterpart.
Assesment of expression PD-1 [receptor] on TILs, which is often overlooked may also be informative. One study found that a PD-L1 high and PD-1 low pattern predicted $86 \%$ of responders to ICI [24]. CD4[+] T cells. Most studies did not confirm that the CD4+ cells had any impact on progression free survival [PFS] or OS in NSCLC $[19,25]$. This may be because CD4+ cells include very distinct cell phenotypes: $\mathrm{T}$ helper lymphocytes [Th1,2,7] and $\mathrm{T}$ regulatory cells [Treg]. Th1 subtype is responsible for polarizing the immune response towards antitumour cell-mediated immunity with CD8+ cells as main effectors. One of the early trials with PD-1 inhibitor found that responses were positively correlated with an increase in Th1 gene expression [26]. More studies on CD4+ and their subtypes in NSCLC are needed to better determine their value as biomarkers. Regulatory $\mathrm{T}$ lymphocytes [Treg] are major suppressors of the adaptive immunity. Treg are CD4+CD25+ cells. These cells are usually detected in IHC by the specific transcription factor FOXP3. These cells are crucial for maintaining peripheral self-tolerance.

The immunosuppressive role of Treg is established early on in cancerogenesis [27]. It was shown that treatment of mice with early NSCLC with antiCD25 antibodies increased the CD8+ infiltration of the tumour, although failed to do so in later stages. However, in advanced stages treatment with antiCD25 significantly increased the response rate [RR] to carboplatin. Treg in tumour tissues negatively influence the prognosis of NSCLC patients. Flow cytometry analysis of the NSCLC showed that infiltrating Treg have a unique expression profile of PD-L1, CCR8 and IL2R, which renders them more immunosuppressive. Also, the expression of Treg cell signature genes, such as LAYN, MAGEH1, or CCR8 in whole-tumour samples correlates with poor prognosis [28]. One study found that the density of FOXP3 + but not CD8+ was a negative predictive factor for response to induction chemoradiotherapy in stage II and III NSCLC [29], which may emphasise their role in early development of immune evasion. Expression of FOXP3 is sometimes also observed in tumour cells. Tao et al. [30] found that by itself the TC FOXP3+ had no prognostic impact.

However, the combination of high density of FOXP3+ TILs and absent FOXP3+ TCs allowed to identify patients with high risk for recurrence after surgery. In a metanalysis the FOXP3 density in tumour stroma appeared to negatively influence both OS and PFS [19]. These results are supported by findings in other cancer types. In $\mathrm{BC}$ high $\mathrm{CD} 8+/ \mathrm{FOXP} 3+$ ratio was an independent prognostic factor for BC specific survival in residual TNBC after neoadjuvant treatment [31].Taken together the results of recent studies suggest that the prognostic and predictive value of TILs may be increased if several cell types are assessed together. This makes sense as the interaction between the CTL, Th and Treg is crucial for the development of the adaptive immune response against cancer. The prognostic effect of these cells may be additionally increased when they are analysed in the context of immune checkpoints and other factors. Tumour associated macrophages [TAMs] sometimes comprise up to $50 \%$ of the tumour tissue [32-34]. They may be activated to become potent tumour suppressors [classically 
activated, M1] or effective tumour promoters [alternatively activated, M2].

Arguably such classification oversimplifies the real-life continuum of TAM phenotypes. Current data shows that TAM polarization is driven by the TME and M2-like TAMs may specialize in promoting angiogenesis [35], providing growth factor support, immunosuppression or supporting invasive growth [36] and contribute to any other malignant hallmark [37]. However, M1/M2 polarization view has been accepted as a practical classification to separate antitumor TAMs from tumour promoting TAMs in clinical studies. Nonetheless there is no standardized method for TAM phenotype assessment in IHC. The most widely employed method is the double staining with general macrophage marker CD68 and another marker specific for M1 or M2. The most commonly used M1 marker is HLA-DR. Other less explored markers include transcription factors STAT1 and IRF5 associated with INF $\gamma$ mediated signalling [36,38]. Meanwhile the variety of used M2 markers is much bigger. Usually scavenger receptors are utilized, the most frequently used are: CD163, CD204, CD206 or CD209 [39].

Majority of the available data shows that TAMs are a significant prognostic factor in NSCLC. Welsh et al demonstrated that tumour islet TAM density and tumour islet/stroma TAM ratio were independent prognostic factors in surgically resected NSCLC patients. Meanwhile stromal TAM density was negatively associated with OS. Moreover, patients with high islet density and incomplete resection survived significantly longer that patients with low density and R0 resection [40]. Ohri et al. were the first group to use additional markers to specify the phenotype of the TAMs. CD68 as a general marker and HLA-DR for M1 and CD163 for M2 TAMs were used. TAM composition in extended and poor survival groups was then compared. Significantly higher M1 density in tumour islets and stroma were observed in the extended survival group, while the poor survival group had higher M2 stromal density [41]. Studies show that in most cases about $95 \%$ of TAMs were in the tumour stroma and co-stained with M2 marker CD163 [42]. This may indicate the crucial role of M2 TAMs in acquisition of malignant hallmarks and explain the predictive value of TAMs in EGFR TKI treated patients. High stromal M2 density and EGFR status were both independent predictive factors in the EGFR TKI treated patients in one study [40].

The available data on TAMs in NSCLC was analysed in a metanalysis. 20 studies from 1999 to 2014 were included. The results showed that high islet CD68+ density, islet M1 density were positively associated with OS, while M2 stromal density was associated with poor OS [43]. However only 4 studies used more than 1 TAM marker and of these 3 used markers for both M1 and M2 TAMs [41,44-46]. Therefore, a lot of unanswered questions remain in this field.

\section{Immune Checkpoints as Biomarkers}

The immune checkpoints are a large group of molecules expressed on the surface of the immune, cancer and cancer stroma cells. Their role is to regulate the extent of the immune response, so it does not become harmful to the host. Cancer cells may express them either as do normal cells - in response to an ongoing immune response or may induce the expression through other pathways that evolve during cancer progression. They are targets of greatly successful immune checkpoint inhibitors [ICI], which are now used for the treatment of NSCLC. Naturally this has been accompanied by the research of IC as predictive biomarkers. However, their value as markers may also extend beyond treatment with ICI. Here we discuss those checkpoints that show prognostic, predictive value and present as therapeutic targets in NSCLC. TIM- 3 is a Th1 and CD8+ co-inhibiting receptor. It serves specifically to limit the duration and extent of Th1 and CTL driven immune responses. The ligand for TIM-3 is galectin- 9 and its role in cancerogenesis is ambiguous. Galactin-9 may be involved in immune suppression through TIM-3, but also can promote cancer cell aggregation, modulate adhesion to ECM $[47,48]$ limit invasion into tissues and limit hematogenic spread $[49,50]$.

In hepatocellular carcinoma it was also shown to induce apoptosis and inhibit cell growth [51]. A metanalysis that sought to asses galectin- 9 prognostic value found it to be positively associated with survival in multiple solid cancers including NSCLC [52]. Because of this multifaceted role of Galectin-9, TIM-3 expression may be more informative in describing immune status of tumours. Preclinical studies with solid and haematological tumour models showed that the most suppressed CD8 lymphocytes express TIM-3 $[53,54]$. These cells co-express PD- 1 and fail to produce IL-2, TNF $\alpha$ and INF $\gamma$. This pattern has been observed in a significant fraction of TILs in melanoma, NSCLC and non-Hodgkin lymphoma [55,56]. TIM-3 has also been found to be expressed on a subset of Treg [Foxp3+] lymphocytes. TIM-3+ Tregs constitute approximately $60 \%$ of all Tregs in NSCLC tissue [55]. The higher TIM-3+Treg infiltration is associated with higher nodal stage and advanced disease. High TIM-3 expression in tumour tissue was found to be associated with more advanced disease and poorer OS in NSCLC $[57,58]$.

Since TIM-3 is often co-expressed with PD-1, the prospect of dual blockade has been explored in preclinical models [55]. In PD-1 immunotherapy resistant B16F10 model of melanoma TIM3/PD-1 co-blockade resulted in a significant tumour response compared with either TIM-3 or PD-1 blockade alone. Expression of TIM-3 on Treg cells and co-expression with PD-1, the two major mechanisms of immune evasion in cancer, make it interesting both as an immune biomarker and a target for novel therapies. LAG-3 is another immune checkpoint inhibitor, that seems to be important in immune evasion by cancer cells [59]. LAG-3 is closely related to CD4 - the sequence of its coding gene is $20 \%$ homologous to CD4 [60]. LAG-3 is mostly expressed on TILs of CD4+ [66], CD8+ [61] and regulatory lineages, but not on resting T cells [62]. LAG3 binds to MHCII molecules with higher affinity than CD4 and causes the lymphocytes to become anergic. LAG-3 also associates with the CD3-TCR complex after TCR engagement on CD4[+] and CD8[+] lymphocytes and acts as a coinhibitory receptor [63]. LAG3 and MHC II interaction also negatively affects T cell activation, proliferation and differentiation [64]. 
Soluble form of LAG-3 [sLAG-3] seems to have a distinct mechanism of action and is important in DC maturation [65]. LAG3 is usually co-expressed with other immune inhibitory molecules such as PD-1 and TIM-3 [59]. In preclinical studies it was found that dual PD-1/LAG-3 blockade with antibodies was able to cure most transplantable tumours that were resistant to treatment by a single antibody [59]. LAG-3 has been shown to be important in cancer immune evasion in multiple tumour types including melanoma [66], ovarian [67], colon [68] and other. In surgically resected NSCLC it was found to be expressed on TILs in $25.6 \%$ and frequently coexpressed with PD-1. Double LAG-3/PD-1 negativity of TILs was associated with lower recurrence rates than tumours with TILs that were positive for at least one of these immune checkpoints. It was also found LAG-3 is more frequently expressed on TILs in nonadenocarcinoma histology [69]. LAG-3 inhibitors are now being studied in phase I studies in several solid tumour types.V domain immunoglobulin suppressor of T cell activation [VISTA] is a unique B7 family checkpoint that regulates a broad spectrum of immune responses. VISTA extracellular domain bears homology with PDL1 [70] and was implicated as one of resistance mechanisms to antiPD-1/PD-L1 antibodies.

The "ligand" nature of VISTA was established using immobilized VISTA ectodomain, which inhibits T cell proliferation, IL-2 and INF $\gamma$ production in a dose-dependent manner $[70,71]$. The mechanism by which VISTA regulates $\mathrm{T}$ cell activation is ill describes as the receptor for VISTA and the exact adaptor proteins are yet unknown. Contrary to PD-L1 VISTA is mostly expressed on hematopoietic cells. Under resting conditions, it is expressed on CD1 ${ }^{4+}$ monocytes, neutrophils, myeloid CD11c+ DCs, and $\mathrm{CD}^{4+}$ and $\mathrm{CD}^{8+} \mathrm{T}$ cells [72]. Under inflammatory conditions VISTA expression is induced by $\mathrm{p} 53$ and geno-toxic stress that induces p53 [73]. Its expression may be informative since its expression does not overlap significantly with other immune checkpoints [74]. In murine cancer models VISTA is expressed by CD11+Gr1+ myeloid cells and FoxP3+ regulatory lymphocytes [75]. VISTA was found to be upregulated in several human cancers. In gastric cancer $>80 \%$ of VISTA is expressed on TILs, while expression on tumour cells is seen in $<10 \%$ of cases [76]. VISTA expression correlates with Lauren classification, tumour localization, Epstein-Barr virus infection, KRAS- and PIK3CA mutational status, and PD-L1 expression.

In oral squamous cell carcinoma high VISTA and low CD8 cell infiltration was associated with poor survival [77]. Increased expression of VISTA ant other immune inhibitors [TIM3, LAG3, PD-L1] was suspected to be the mechanism of resistance to immunotherapy in human prostate carcinoma and melanoma following treatment with ipilimumab [78]. Few studies on VISTA expression in NSCLC are available. Villarroel-Espindola et al studied VISTA expression in NSCLC samples from 758 cases by using quantitative immunofluorescence. VISTA expression was present in $99 \%$ of NSCLC cases. VISTA was predominantly expressed on hematopoietic cells in accordance with other studies. VISTA expression positively correlated with PD-L1, PD-1, CD ${ }^{8+}$ T-cells and CD68+ macrophages. The expression was higher in $\mathrm{T}$ cells than in macrophages, it was the highest in CD8+ T-cells.
Tumours with high VISTA expression were predominantly of low tumour mutational burden and EGFR wild type. Presence of VISTA in tumour compartment predicted longer 5-year survival [79].

TIGIT [T-cell immunoglobulin and ITIM domain] is another checkpoint inhibitor receptor expressed mainly on TILs: natural killer cells, effector/memory and regulatory T-cells [80,81]. The ligands for TIGIT are CD155 and CD 112. Both are found to be expressed by tumour cells, APCs and other T-cells [82]. TIGIT competes for CD155 with other stimulatory [CD226] but also inhibitory receptors [CD96] [83-85]. Binding of CD155 by TIGIT results in suppression of IL-12 production and therefore a shift away from Th1 regulated cell- mediated immunity, to a immune-tolerant state dominated by Tregs and Th2 [86]. TIGIT is often expressed along other inhibitory checkpoint proteins like PD-1, TIM3, LAG-3, which results in a exhausted T-cell phenotype [87]. There is also evidence from tumour models for synergistic activity of TIGIT inhibitors when used with either PD-1 or TIM3 inhibitors, which makes TIGIT another potential target for novel immunotherapies and a possible immune biomarker [86-88].

Factors of the tumour microenvironment, IDO. Soluble factors in the TME are another important aspect that influence the anticancer immune response. Their assessment may shed light on immunosuppression exerted on TILs. Arguably one of the most prominent is IDO [indoleamine 2,3-dioxygenase]. IDO is expressed mainly by cancer cells and some MDSC and is secreted into extracellular environment. IDO converts tryptophan [Try] essential to $\mathrm{T}$ cell function into kynurenine [Kyn] [89], causes naive CD4[+] cell conversion to Treg [90] and facilitates MDSC expansion [91]. It may be also feasible to asses IDO activity in tumour tissues by measuring Try/Kyn ratio, which may present as another TME related biomarker [92]. In IDO deficient mice with lung cancer IDO deficiency contributes to MDSC depletion, lower PD-1 expression on CD8[+] TILs and higher expression of INF $\gamma$. This results in lower tumour burden [93]. Preclinical studies show that IDO downregulation induces sensitivity to pemetrexed and gemcitabine [94]. IDO has also been implicated in resistance to CTLA-4 inhibitors in melanoma cell lines [95]. IDO expression in tumour tissue has been demonstrated to be prognostic of overall survival in some tumour types [96]. NSCLC is among the tumours with high IDO activity in their microenvironment [97].

In NSCLC a post-induction chemotherapy increase in IDO activity portends worse OS [98]. These developments lead IDO inhibitors into early phase clinical trials which demonstrated excellent safety, but modest activity with stable disease being the best response [99-101]. This sparked an interest in combining these agents with antiPD-1 antibodies and trials investigating this approach have shown more promising results [102,103].

\section{Conclusion}

It is now evident that any type of cancer represents a failure of the immune system at some stage or the other. NSCLC is now at the leading edge in the development of immunotherapy. Despite some impressive responses, a significant portion of patients progress or 
do not respond to ICIs at all. This situation urges us to seek new markers that will help us define the immunological phenotype of cancer and the individual mechanisms of immune evasion. PD-L1 is the most researched immune marker in NSCLC since it is the direct target of novel immunotherapies. Nonetheless, PD-L1 expression remains much kto be desired as a predictive factor of response to ICI. Many patients with PD-L1 negative tumours experience responses greater than standard second line chemotherapy. Immune cells infiltrating the tumour are the logical candidate for an immune marker, because they conduct the immune response and are the target of immunosuppressive mechanisms. TILs are the main effector cells of the immune response and have been shown to be useful immune markers in NSCLC.

TAMs, depending on their state of activation, may be involved in both immune response and immune evasion. Data from multiple studies supports the value of TAMs as prognostic markers, although standardization of their routine assessment remains a major obstacle. Tregs are the major suppressive cells in NSCLC and have also been shown to be crucial for NSCLC prognosis by multiple studies. In any case the complexity of interactions between cancer cells, cancer stroma and immune cells will likely require comprehensive analysis of several markers, to identify immunosuppressive drivers in daily practice.

\section{Acknowledgement}

The article was written as a part of work in a project "Modern technologies to resolve a complex structure of tumor" being conducted at the National Cancer Institute of Lithuania and lead by PhD Sonata Jarmalaitè.

\section{References}

1. Hanahan D, Weinberg R (2011) Hallmarks of Cancer: The Next Generation. Cell 144(5): 646-674.

2. Mittal D, Gubin M, Schreiber R, Smyth M (2014) New insights into cancer immunoediting and its three component phases-elimination, equilibrium and escape. Current Opinion in Immunology 27: 16-25.

3. Chen DS, Mellman I (2013) Oncology meets immunology: the cancerimmunity cycle. Immunity 39(1): 1-10.

4. Chen DS, Mellman I (2017) Elements of cancer immunity and the cancerimmune set point. Nature 541(7637): 321-330.

5. Galon J, Pagès F, Marincola F (2012) Cancer classification using the Immunoscore: a worldwide task force. Journal of Translational Medicine 10(1): 205.

6. Loi S, Michiels S, Salgado R (2014) Tumor infiltrating lymphocytes are prognostic in triple negative breast cancer and predictive for trastuzumab benefit in early breast cancer: results from the FinHER trial. Annals of Oncology 25(8): 1544-1550.

7. Salgado R, Denkert C, Demaria S (2014) The evaluation of tumorinfiltrating lymphocytes (TILs) in breast cancer: recommendations by an International TILs Working Group 2014. Annals of Oncology 26(2): 259-271.

8. Pruneri G, Vingiani A, Bagnardi V (2015) Clinical validity of tumorinfiltrating lymphocytes analysis in patients with triple-negative breast cancer. Annals of Oncology 27(2): 249-256.

9. Denkert C, von Minckwitz G, Brase J (2015) Tumor-Infiltrating Lymphocytes and Response to Neoadjuvant Chemotherapy With or Without Carboplatin in Human Epidermal Growth Factor Receptor
2-Positive and Triple-Negative Primary Breast Cancers. Journal of Clinical Oncology 33(9): 983-991.

10. Salgado R, Denkert C, Campbell C (2015) Tumor-Infiltrating Lymphocytes and Associations With Pathological Complete Response and Event-Free Survival in HER2-Positive Early-Stage Breast Cancer Treated With Lapatinib and Trastuzumab. JAMA Oncology 1(4): 448.

11. Galon J, Mlecnik B, Marliot F (2016) Validation of the immunoscore (IM) as a prognostic marker in stage I/II/III colon cancer: results of a worldwide consortium-based analysis of 1,336 patients. J Clin Oncol 34: 3500 .

12. Wang L, Zhai Z, Ji D, Li Z, Gu J, et al. (2015) Prognostic value of CD45RO(+) tumor-infiltrating lymphocytes for locally advanced rectal cancer following $30 \mathrm{~Gy} / 10 \mathrm{f}$ neoadjuvant radiotherapy. International Journal of Colorectal Disease 30(6): 753-760.

13. Brambilla E, Le Teuff G, Marguet S (2016) Prognostic Effect of Tumor Lymphocytic Infiltration in Resectable Non-Small-Cell Lung Cancer. Journal of Clinical Oncology 34(11): 1223-1230.

14. Donnem T, Hald S, Paulsen E (2015) Stromal CD8+ T-cell Density-A Promising Supplement to TNM Staging in Non-Small Cell Lung Cancer. Clinical Cancer Research 21(11): 2635-2643.

15. Goc J, Germain C, Vo Bourgais TK (2014) Dendritic cells in tumorassociated tertiary lymphoid structures signal a Th1 cytotoxic immune contexture and license the positive prognostic value of infiltrating CD8+ T cells. Cancer Res 74: 705-715.

16. Schalper KA, Brown J, Carvajal Hausdorf D (2015) Objective measurement and clinical significance of TILs in non-small cell lung cancer. J Natl Cancer Inst 3: 107.

17. Paulsen E, Kilvaer T, Khanehkenari M (2015) CD45RO+ Memory T Lymphocytes-a Candidate Marker for TNM-Immunoscore in Squamous Non-Small Cell Lung Cancer. Neoplasia 17(11): 839-848.

18. Liu H, Zhang T, Ye J (2012) Tumor-infiltrating lymphocytes predict response to chemotherapy in patients with advance non-small cell lung cancer. Cancer Immunology, Immunotherapy 61(10): 1849-1856.

19. Geng Y, Shao Y, He W (2015) Prognostic Role of Tumor-Infiltrating Lymphocytes in Lung Cancer: a Meta-Analysis. Cellular Physiology and Biochemistry 37(4): 1560-1571.

20. Wang L, Wu Z, Zhang W (2015) Prognostic Significance of Tumorinfiltrating CD8+or CD3+T Lymphocytes and Interleukin-2 Expression in Radically Resected Non-small Cell Lung Cancer. Chinese Medical Journal 128(1): 105.

21. Djenidi F, Adam J, Goubar AV (2015) CD8+CD103+Tumor-Infiltrating Lymphocytes Are Tumor-Specific Tissue-Resident Memory T Cells and a Prognostic Factor for Survival in Lung Cancer Patients. The Journal of Immunology 194(7): 3475-3486.

22. Aguilar Cazares D, Meneses Flores M, Prado Garcia H (2014) Relationship of Dendritic Cell Density, HMGB1 Expression, and Tumorinfiltrating Lymphocytes in Non-Small Cell Lung Carcinomas. Applied Immunohistochemistry \& Molecular Morphology 22(2): 105-113.

23. Mazzaschi G, Madeddu D, Bocchialini G (2017) 20Favorable clinical outcome and response to immunotherapy share a common PD-L1/PD-1 based NSCLC immune contexture. Annals of Oncology: pp. 28.

24. Tokito T, Azuma K, Kawahara A (2016) Predictive relevance of PD-L1 expression combined with CD8+ TIL density in stage III non-small cell lung cancer patients receiving concurrent chemoradiotherapy. European Journal of Cancer 55: 7-14.

25. Sterlacci W, Wolf D, Savic S (2012) High transforming growth factor beta expression represents an important prognostic parameter for surgically resected non-small cell lung cancer. Hum Pathol 43: 339-349.

26. Herbst R, Soria J, Kowanetz M (2014) Predictive correlates of response to the anti-PD-L1 antibody MPDL3280A in cancer patients. Nature 515(7528): 563-567. 
27. Ganesan A, Johansson M, Ruffell B (2013) Tumor-Infiltrating Regulatory $\mathrm{T}$ Cells Inhibit Endogenous Cytotoxic $\mathrm{T}$ Cell Responses to Lung Adenocarcinoma. The Journal of Immunology 191(4): 2009-2017.

28. De Simone M, Arrigoni A, Rossetti G (2016) Transcriptional Landscape of Human Tissue Lymphocytes Unveils Uniqueness of Tumor-Infiltrating T Regulatory Cells. Immunity 45(5): 1135-1147.

29. Tao H, Shien K, Soh J (2014) Density of Tumor-Infiltrating FOXP3+ T Cells as a Response Marker for Induction Chemoradiotherapy and a Potential Prognostic Factor in Patients Treated with Trimodality Therapy for Locally Advanced Non-Small Cell Lung Cancer. Annals of Thoracic and Cardiovascular Surgery 20(6): 980-986.

30. Tao H, Mimura Y, Aoe K (2012) Prognostic potential of FOXP3 expression in non-small cell lung cancer cells combined with tumor-infiltrating regulatory T cells. Lung Cancer 75(1): 95-101.

31. Miyashita M, Sasano H, Tamaki K (2015) Prognostic significance of tumor-infiltrating CD8+ and FOXP3+ lymphocytes in residual tumors and alterations in these parameters after neoadjuvant chemotherapy in triple-negative breast cancer: a retrospective multicenter study. Breast Cancer Research 17(1): 124.

32. Van Overmeire E, Laoui D, Keirsse J, Van Ginderachter JA, Sarukhan A, et al. (2014) Mechanisms driving macrophage diversity and specialization in distinct tumor microenvironments and parallelisms with other tissues. Frontiers in Immunology 5: 127.

33. Tu E, Chia PZC, Chen W (2014) TGF $\beta$ in T cell biology and tumor immunity: angel or devil? Cytokine and Growth Factor Reviews 25(4): 423-435.

34. Kelly PMA, Davison RS, Bliss E, McGee JO (1988) Macrophages in human breast disease: a quantitative immunohistochemical study. British Journal of Cancer 57(2): 174-177.

35. Riabov V, Gudima A, Wang N, Mickley A, Orekhov A, et al. (2014) Role of tumor associated macrophages in tumor angiogenesis and lymphangiogenesis. Frontiers in Physiology 5: 75.

36. Williams C, Yeh E, Soloff A (2016) Tumor-associated macrophages: unwitting accomplices in breast cancer malignancy. npj Breast Cancer 2(1): 15.

37. Mantovani A, Marchesi F, Malesci A, Laghi L, Allavena P, et al. (2017) Tumour-associated macrophages as treatment targets in oncology. Nature Reviews Clinical Oncology 14(7): 399-416.

38. Tymoszuk P, Evens H, Marzola V (2014) In situ proliferation contributes to accumulation of tumor-associated macrophages in spontaneous mammary tumors. European Journal of Immunology 44(8): 2247-2262.

39. Zhang Q, Liu L, Gong C (2012) Prognostic Significance of TumorAssociated Macrophages in Solid Tumor: A Meta-Analysis of the Literature. PLoS ONE 7(12): e50946.

40. Welsh T, Green R, Richardson D, Waller D, O’Byrne K, et al. (2005) Macrophage and Mast-Cell Invasion of Tumor Cell Islets Confers a Marked Survival Advantage in Non-Small-Cell Lung Cancer. Journal of Clinical Oncology 23(35): 8959-8967.

41. Ohri C, Shikotra A, Green R, Waller D, Bradding P, et al. (2009) Macrophages within NSCLC tumour islets are predominantly of a cytotoxic M1 phenotype associated with extended survival. European Respiratory Journal 33(1): 118-126.

42. Chung F, Lee K, Wang C (2012) Tumor-associated macrophages correlate with response to epidermal growth factor receptor-tyrosine kinase inhibitors in advanced non-small cell lung cancer. International Journal of Cancer 131(3): E227-E235.

43. Mei J, Xiao Z, Guo C (2016) Prognostic impact of tumor-associated macrophage infiltration in non-small cell lung cancer: A systemic review and meta-analysis. Oncotarget $7(23)$.

44. Zhang BC, Yao GQ Zhang YF (2011) M2-Polarized tumor-associated macrophages are associated with poor prognoses resulting from accelerated lymphangiogenesis in lung adenocarcinoma. Clinics 66: 1879-1886.

45. Ohtaki Y, Ishii G, Nagai K (2010) Stromal macrophage expressing CD204 is associated with tumor aggressiveness in lung adenocarcinoma. J Thorac Oncol 5: 1507-1515.

46. Ma J, Liu L, Che G (2010) The M1 form of tumor-associated macrophages in non-small cell lung cancer is positively associated with survival time. BMC Cancer 10: 112.

47. Kasamatsu A., Uzawa K., Nakashima D (2005) Galectin-9 as a regulator of cellular adhesion in human oral squamous cell carcinoma cell lines. Int J Mol Med 16: 269-273.

48. Zhang F, Zheng M, Qu Y (2009) Different roles of galectin-9 isoforms in modulating E-selectin expression and adhesion function in LoVo colon carcinoma cells. Mol Biol Rep 36: 823-830.

49. Irie A, Yamauchi A, Kontani K, Kihara M (2005) Galectin-9 as a prognostic factor with antimetastatic potential in breast cancer. Clin Cancer Res 11: 29620-2968.

50. Yamauchi A, Kontani K, Kihara M, Nishi N, Yokomise H, et al. (2006) Galectin-9, a novel prognostic factor with antimetastatic potential in breast cancer. Breast J 12(5): S196-S200.

51. Fujita K, Iwama H, Sakamoto T (2015) Galectin-9 suppresses the growth of hepatocellular carcinoma via apoptosis in vitro and in vivo. Int J Oncol 46: 2419-2430.

52. Zhou X, Sun L, Jing D (2018) Galectin-9 Expression Predicts Favorable Clinical Outcome in Solid Tumors: A Systematic Review and MetaAnalysis. Front Physiol 9: 452.

53. Sakuishi K, Apetoh L, Sullivan J, Blazar B, Kuchroo V, et al. (2011) Targeting Tim-3 and PD-1 pathways to reverse T cell exhaustion and restore anti-tumor immunity. The Journal of Experimental Medicine 208(6): 1331-1331.

54. Zhou Q, Munger M, Veenstra R (2011) Coexpression of Tim-3 and PD-1 identifies a CD8+ T-cell exhaustion phenotype in mice with disseminated acute myelogenous leukemia. Blood 117(17): 4501-4510.

55. Gao X, Zhu Y, Li G (2012) TIM-3 Expression Characterizes Regulatory T Cells in Tumor Tissues and Is Associated with Lung Cancer Progression. PLoS ONE 7(2): e30676.

56. Yang Z, Grote D, Ziesmer S (2012) IL-12 upregulates TIM-3 expression and induces $\mathrm{T}$ cell exhaustion in patients with follicular B cell nonHodgkin lymphoma. Journal of Clinical Investigation 122(4): 1271-1282.

57. Xu LY, Chen DD, He JY (2014) Tim-3 expression by peripheral natural killer cells and natural killer $\mathrm{T}$ cells increases in patients with lung cancer-reduction after surgical resection. Asian Pac J Cancer Prev 15: 9945-9948.

58. Xu L, Huang Y, Tan L (2015) Increased Tim-3 expression in peripheral NK cells predicts a poorer prognosis and Tim-3 blockade improves NK cell-mediated cytotoxicity in human lung adenocarcinoma. Int Immunopharmacol 29: 635-641.

59. Woo S, Turnis M, Goldberg M (2011) Immune Inhibitory Molecules LAG3 and PD-1 Synergistically Regulate T-cell Function to Promote Tumoral Immune Escape. Cancer Research 72(4): 917-927.

60. Carlo E, Cappello P, Sorrentino C (2005) Immunological mechanisms elicited at the tumour site by lymphocyte activation gene-3 (LAG-3) versus IL-12: sharing a common Th1 anti-tumour immune pathway. The Journal of Pathology 205(1): 82.

61. Grosso J, Kelleher C, Harris T (2007) LAG-3 regulates CD8+ T cell accumulation and effector function in murine self- and tumor-tolerance systems. Journal of Clinical Investigation 117(11): 3383-3392.

62. Shih K, Arkenau H, Infante J (2014) Clinical Impact of Checkpoint Inhibitors as Novel Cancer Therapies. Drugs 74(17): 1993-2013.

63. Hannier S, Triebel F (1999) The MHC class II ligand lymphocyte 
activation gene-3 is co-distributed with CD8 and CD3-TCR molecules after their engagement by mAb or peptide-MHC class I complexes International Immunology 11(11): 1745-1752.

64. Huard B, Prigent P, Pagès F, Bruniquel D, Triebel F, et al. (1996) T cell major histocompatibility complex class II molecules down-regulate CD4+ T cell clone responses following LAG-3 binding. European Journal of Immunology 26(5): 1180-1186.

65. Andreae S (2003) MHC class II signal transduction in human dendritic cells induced by a natural ligand, the LAG-3 protein (CD223). Blood 102(6): 2130-2137.

66. Hemon P, Jean Louis F, Ramgolam K (2011) MHC Class II Engagement by Its Ligand LAG-3 (CD223) Contributes to Melanoma Resistance to Apoptosis. The Journal of Immunology 186(9): 5173-5183.

67. Matsuzaki J, Gnjatic S, Mhawech Fauceglia P (2010) Tumor-infiltrating NY-ESO-1-specific CD8+T cells are negatively regulated by LAG-3 and PD-1 in human ovarian cancer. Proceedings of the National Academy of Sciences 107(17): 7875-7880.

68. Losa N, Cruise M, Tam A (2015) The vigorous immune microenvironment of microsatellite instable colon cancer is balanced by multiple counterinhibitory checkpoints. Journal for ImmunoTherapy of Cancer 3(2): 410

69. He Y, Yu H, Rozeboom L (2017) LAG-3 Protein Expression in Non-Small Cell Lung Cancer and Its Relationship with PD-1/PD-L1 and TumorInfiltrating Lymphocytes. J Thorac Oncol 12(5): 814-823.

70. Wang L, Rubinstein R, Lines JL (2011) VISTA, a novel mouse Ig superfamily ligand that negatively regulates $\mathrm{T}$ cell responses. J Exp Med 208: 577-592.

71. Lines JL, Pantazi E, Mak J (2014) VISTA Is an Immune Checkpoint Molecule for Human T Cells. Cancer Res 74: 1924-1932.

72. Bharaj P, Chahar HS, Alozie OK (2014) Characterization of programmed death-1 homologue-1 (PD-1H) expression and function in normal and HIV infected individuals. PLoS One 9: e109103.

73. Yoon KW, Byun S, Kwon E (2015) Control of signaling-mediated clearance of apoptotic cells by the tumor suppressor. Science 349: 1261669.

74. Villanueva N, Bazhenova L (2018) New strategies in immunotherapy for lung cancer: beyond PD-1/PD-L1. Ther Adv Respir Dis 12: 1753466618794133

75. Le Mercier I, Chen W, Lines JL (2014) VISTA Regulates the Development of Protective Antitumor Immunity. Cancer Res 74: 1933-1944.

76. Boger C, Behrens HM, Kruger S, Rocken C (2017) The novel negative checkpoint regulator VISTA is expressed in gastric carcinoma and associated with PD-L1/PD-1: A future perspective for a combined gastric cancer therapy? Oncoimmunology 6: e1293215.

77. Wu L, Deng WW, Huang CF (2017) Expression of VISTA correlated with immunosuppression and synergized with CD8 to predict survival in human oral squamous cell carcinoma. Cancer Immunol Immunother 66: 627-636.

78. Gao J, Ward JF, Pettaway CA (2017) VISTA is an inhibitory immune checkpoint that is increased after ipilimumab therapy in patients with prostate cancer. Nat Med 23: 551-555.

79. Villarroel Espindola F, Yu X, Datar N (2018) Spatially resolved and quantitative analysis of VISTA/PD-1H as a novel immunotherapy target in human non-small cell lung cancer. Clin Cancer Res 24(7): 1562-1573.

80. Stanietsky N, Simic H, Arapovic J (2009) The interaction of TIGIT with PVR and PVRL2 inhibits human NK cell cytotoxicity. Proc Natl Acad Sci USA 106: 17858-17863.

81. Yu X, Harden K, Gonzalez LC (2009) The surface protein TIGIT suppresses $\mathrm{T}$ cell activation by promoting the generation of mature immunoregulatory dendritic cells. Nat Immunol 10: 48-57.

82. Anderson AC, Joller N, Kuchroo VK (2016) Lag-3, Tim-3, and TIGIT: co- inhibitory receptors with specialized functions in immune regulation. Immunity 44: 989-1004.

83. Chan CJ, Andrews DM, McLaughlin NM (2010) DNAM-1/CD155 interactions promote cytokine and NK cell-mediated suppression of poorly immunogenic melanoma metastases. J Immunol 184: 902-911.

84. Chan CJ, Andrews DM, Smyth MJ (2012) Receptors that interact with nectin and nectinlike proteins in the immunosurveillance and immunotherapy of cancer. Curr Opin Immunol 24: 246-251.

85. Chan CJ, Martinet L, Gilfillan S (2014) The receptors CD96 and CD226 oppose each other in the regulation of natural killer cell functions. Nat Immunol 15: 431-438.

86. Kurtulus S, Sakuishi K, Ngiow SF (2015) TIGIT predominantly regulates the immune response via regulatory T cells. J Clin Invest 125: 40534062 .

87. Johnston RJ, Comps Agrar L, Hackney J (2014) The immunoreceptor TIGIT regulates antitumor and antiviral CD8(+) T cell effector function. Cancer Cell 26: 923-937.

88. Chauvin JM, Pagliano O, Fourcade J (2015) TIGIT and PD-1 impair tumor antigen-specific CD8+ T cells in melanoma patients. J Clin Invest 125 : 2046-2058.

89. Platten M, Wick W, Van den Eynde B (2012) Tryptophan Catabolism in Cancer: Beyond IDO and Tryptophan Depletion. Cancer Research 72(21): 5435-5440.

90. Wang R, Green D (2012) Metabolic reprogramming and metabolic dependency in T cells. Immunological Reviews 249(1): 14-26.

91. Smith C, Chang M, Parker K (2012) IDO Is a Nodal Pathogenic Driver of Lung Cancer and Metastasis Development. Cancer Discovery 2(8): 722735.

92. Brochez L, Chevolet I, Kruse V (2017) The rationale of indoleamine 2,3-dioxygenase inhibition for cancer therapy. Eur J Cancer 76: 167-182.

93. Schafer C, Wang Y, Hough K (2016) Indoleamine 2,3-dioxygenase regulates anti-tumor immunity in lung cancer by metabolic reprogramming of immune cells in the tumor microenvironment. Oncotarget 7(46).

94. Maleki Vareki S, Chen D, Di Cresce C (2015) IDO Downregulation Induces Sensitivity to Pemetrexed, Gemcitabine, FK866, and Methoxyamine in Human Cancer Cells. PLOS ONE 10(11): e0143435.

95. Holmgaard R, Zamarin D, Munn D, Wolchok J, Allison J, et al. (2013) Indoleamine 2,3-dioxygenase is a critical resistance mechanism in antitumor $\mathrm{T}$ cell immunotherapy targeting CTLA-4. The Journal of Experimental Medicine 210(7): 1389-1402.

96. Jia Y, Wang H, Wang Y (2015) Low expression of Bin1, along with high expression of IDO in tumor tissue and draining lymph nodes, are predictors of poor prognosis for esophageal squamous cell cancer patients. International Journal of Cancer 137(5): 1095-1106.

97. Théate I, van Baren N, Pilotte L (2015) Extensive profiling of the expression of the indoleamine 2,3-dioxygenase 1 protein in normal and tumoral human tissues. Cancer Immunol Res 3: 161-172.

98. Creelan B, Bepler G, Antonia S, Garrett T, Soliman H (2011) Indoleamine 2,3-dioxygenase activity and clinical outcome following induction chemotherapy and concurrent chemoradiation in stage III non-small cell lung cancer. Journal of Clinical Oncology 29(15): 10538-10538.

99. Iversen T, Engell Noerregaard L, Ellebaek (2013) Long-lasting Disease Stabilization in the Absence of Toxicity in Metastatic Lung Cancer Patients Vaccinated with an Epitope Derived from Indoleamine 2,3 Dioxygenase. Clinical Cancer Research 20(1): 221-232.

100. Beatty GL, O’Dwyer PJ, Clark J (2017) First in-human phase I study of the oral inhibitor of indoleamine 2,3-dioxygenase-1 epacadostat (INCB024360) in patients with advanced solid malignancies. Clin Cancer Res 23: 3269-3276. 
101. Chevolet I, Speeckaert R, Schreuer M (2015) Characterization of the in vivo immune network of IDO, tryptophan metabolism, PD-L1, and CTLA-4 in circulating immune cells in melanoma. Oncoimmunology 4: e982382.

102. Gangadhar T, Schneider B, Bauer T (2017) Efficacy and safety of epacadostat plus pembrolizumab treatment of NSCLC: preliminary

ISSN: 2574-1241

DOI: 10.26717/BJSTR.2019.12.002325

JB Jaroslav Bublevič. Biomed J Sci \& Tech Res

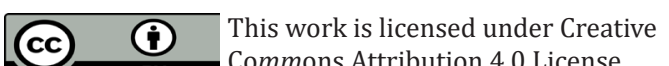

Submission Link: https://biomedres.us/submit-manuscript.php phase I/II results of ECHO-202/KEYNOTE-037. J Clin Oncol 35: 9014.

103. Long GV, Dummer R, Hamid (2018) Epacadostat (E) plus pembrolizumab (P) versus pembrolizumab alone in patients (pts) with unresectable or metastatic melanoma: results of the phase 3 ECHO-301/ KEYNOTE-252 study. J Clin Oncol 36: 108.

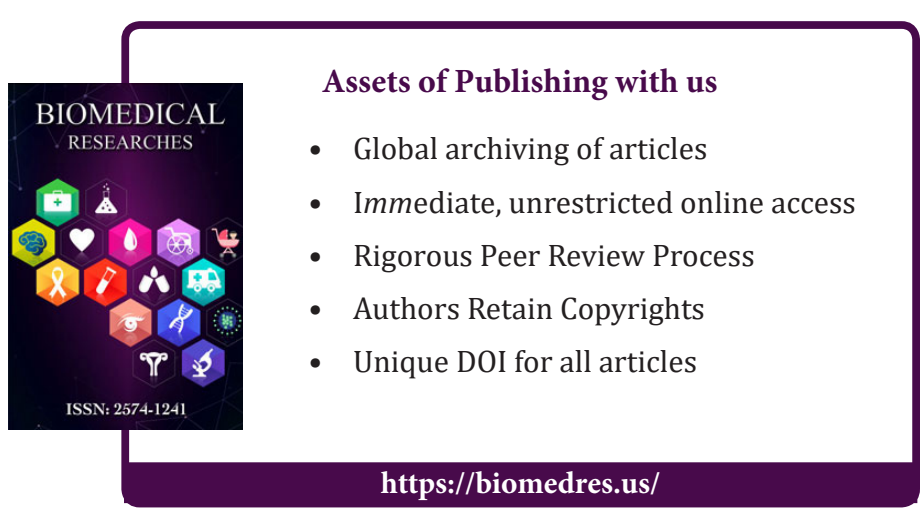

